\title{
Editorial Commentary: Tissue factor expression by the endothelium: Coagulation or inflammation?
}

\author{
Silvio Antoniak, $\mathrm{PhD}^{a, b, *}$, and Nigel Mackman, $\mathrm{PhD}^{a}$ \\ ${ }^{a}$ Department of Medicine, UNC McAllister Heart Institute, University of North Carolina at Chapel Hill, Chapel Hill, NC \\ ${ }^{b}$ Department of Pathology and Laboratory Medicine, University of North Carolina at Chapel Hill, Chapel Hill, NC
}

In this issue of Trends in Cardiovascular Medicine, Witkowski et al. [1] review studies proposing that tissue factor (TF) links coagulation and inflammation. Importantly, TF is a cofactor for the coagulation protease factor VIIa (FVIIa) and therefore it is the TF:FVIIa complex that initiates the coagulation cascade. Interestingly, it has structural homology to members of the class 2 cytokine receptor family. The primary role of the TF:FVIIa complex is to maintain hemostasis. Indeed, a complete deficiency of either TF or FVII is not compatible with life [2]. Activation of the coagulation cascade by the TF: FVIIa complex generates a number of serine proteases that can activate cells and enhance inflammation by cleavage of protease activated receptors (PARs). For instance, FXa activates PAR2 and thrombin activates PAR1. Therefore, the TF: FVIIa complex has a secondary role as an enhancer of inflammation.

Witkowski et al. [1] propose that full length TF expression by endothelial cells plays a central role in both coagulation and inflammation. What is the evidence to support this proposal? Bevilacqua et al. [3] reported that IL-1 $\beta$ induces TF expression in cultured human umbilical endothelial cells (HUVECs). Since HUVECs are relatively easy to isolate, this study opened the flood gates for numerous studies and therefore many studies are done with this cultured cell model. Indeed, a multitude of studies on TF expression in endothelial cells (ECs) were performed [4]. In contrast to the in vitro studies, there is a paucity of evidence that convincingly shows that TF is expressed by the endothelium in vivo [2]. This is due to several reasons: the difficulty in detecting low levels of TF expression on the endothelium using immunohistochemistry, distinguishing TF expression by the endothelium from TF expression by cells within the vessel wall, and distinguishing TF expression from the endothelium itself from the deposition of TF-positive extracellular vesicles derived from other cells, such as monocytes.

We have used a genetic approach to determine the role of $\mathrm{TF}$ in different cell types on coagulation and inflammation in different mouse disease models. First, we used mice that express low levels of TF in all tissues, so-called low TF mice [5]. Secondly, we used $\mathrm{TF}^{\mathrm{fl} / \mathrm{fl}}$ mice that have the $\mathrm{TF}$ gene deleted in a cell type-specific manner [6]. For instance, $\mathrm{TF}^{\mathrm{f} / \mathrm{fl}}$, Tie2Cre mice have TF deleted in both hematopoietic and ECs. We will discuss the role of TF in different cell types in three mouse models: carotid artery thrombosis, endotoxemia, and sickle cell disease.

TF contributes to various forms of thrombosis, particularly atherothrombosis [7]. In the normal blood vessel wall TF is absent in the endothelium, "variably present in cells composing the media" and highly expressed by adventitial cells [8]. Low TF mice exhibited reduced thrombosis in a Rose Bengal model of carotid artery injury [9]. Bone marrow transplant experiments showed that TF expression by nonhematopoietic cells drives thrombosis in this model. Furthermore, deletion of $\mathrm{TF}$ in vascular smooth muscle cells $\left(\mathrm{TF}^{\mathrm{f} / \mathrm{fl}}\right.$, SM22 $\alpha$ Cre mice) reduced thrombosis in a ferric chloride model of carotid artery injury [10]. It should be noted that expression of the Cre recombinase from the SM22 $\alpha$ promoter also reduces TF expression in some adventitial fibroblasts. As expected, deletion of TF in both ECs and hematopoietic cells did not affect thrombosis in the ferric chloride model [11]. These results indicate that TF expression by vascular smooth muscle cells and possibly advential fibroblasts but not

The authors have indicated that there are no conflicts of interest.

*Corresponding author. Tel.: +1 919843 7305; fax: +1 9198434896.

E-mail address: antoniak@email.unc.edu (S. Antoniak). 
endothelial cells drives thrombosis after injury of the mouse carotid artery.

TF plays a central role in the activation of coagulation in animal models of acute endotoxemia and sepsis [12]. In vitro studies showed that LPS induces TF expression in human monocytes and we showed that LPS induces TF expression in HUVECs [4]. Therefore, monocytes and the endothelium are the two most likely sources of TF expression that activate coagulation during endotoxemia. Interestingly, inhibition of $\mathrm{TF}$ in endotoxemic chimpanzees reduced coagulation but did not reduced TNF $\alpha$ or IL- 6 expression [12]. We found that low TF mice had reduced activation of coagulation and a small reduction IL-6 expression but not change in TNF $\alpha$ expression [12]. Similar to low TF mice, we found that mice with TF deleted in both endothelial cells and hematopoietic cells ( $\mathrm{TF}^{\mathrm{f} / \mathrm{fl}}$,Tie2Cre) had reduced activation of coagulation [6]. We then performed bone marrow transplantation experiments to specifically determine the role of TF expression by either hematopoietic cells or ECs on the activation of coagulation. As expected, we found that deletion of TF in hematopoietic cells reduced coagulation in endotoxemic mice. Surprisingly, however, deletion of TF in ECs did not affect the activation of coagulation after administration of LPS [6]. These data indicated that TF expression of TF in ECs in vivo did not contribute to the activation of coagulation in a mouse endotoxemia model.

We have also analyzed the contribution of TF to the activation of coagulation and inflammation in a mouse model of sickle cell disease. This chronic model involves transplantation of bone marrow from sickle mice into recipient mice and takes 5 months to generate a phenotype. First, we showed that inhibition of all sources of TF reduced coagulation, as well as IL-6 and soluble VCAM-1 expression [13]. Next, we transplanted sickle bone marrow into $\mathrm{TF}^{\mathrm{f} / \mathrm{fl}}$, Tie2Cre that have a deficiency of TF in ECs. Consistent with the endotoxemia study, deletion of TF in endothelial cells did not affect activation of coagulation in sickle cell mice [13]. However, a deficiency of TF in endothelial cells did significantly reduce IL-6 expression but not soluble VCAM-1 expression. In a subsequent study, we found that a deficiency of PAR2 or treatment of wild-type mice with the FXa inhibitor rivaroxaban also reduced IL-6 expression [14]. These studies suggest that formation of a TF:FVIIa complex on the endothelial surface generates FXa that cleaves PAR2 to increase IL-6 expression.

In conclusion, despite the numerous in vitro studies showing TF expression in cultured ECs, genetic studies using mouse models indicate that (1) TF expression by vascular smooth muscle cells and adventitial fibroblasts but not ECs or hematopoietic cells drives carotid artery thrombosis, (2) TF expression by hematopoietic cells and adventitial cells but not ECs activates coagulation in endotoxemia, (3) TF expression by hematopoietic cells and adventitial cells but not ECs activates coagulation in sickle cell disease, and (4) EC $\mathrm{TF}$ but not hematopoietic cell or adventitial cell TF contributes to IL-6 expression in sickle cell disease.

\section{R E F E R E N C E S}

[1] Witkowski M, Landmesser U, Rauch U. Tissue factor as a link between inflammation and coagulation. Trends Cardiovasc Med 2016, http://dx.doi.org/10.1016/j.tcm.2015.12.001.

[2] Mackman N. Tissue-specific hemostasis in mice. Arterioscler Thromb Vasc Biol 2005;25:2273-81.

[3] Bevilacqua MP, Pober JS, Majeau GR, Cotran RS, Gimbrone MA Jr. Interleukin 1 (IL-1) induces biosynthesis and cell surface expression of procoagulant activity in human vascular endothelial cells. J Exp Med 1984;160:618-23.

[4] Bode M, Mackman N. Regulation of tissue factor gene expression in monocytes and endothelial cells: thromboxane A2 as a new player. Vascul Pharmacol 2014;62:57-62.

[5] Parry GC, Erlich JH, Carmeliet P, Luther T, Mackman N. Low levels of tissue factor are compatible with development and hemostasis in mice. J Clin Invest 1998;101:560-9.

[6] Pawlinski R, Wang JG, Owens AP 3rd, Williams J, Antoniak S, Tencati M, et al. Hematopoietic and nonhematopoietic cell tissue factor activates the coagulation cascade in endotoxemic mice. Blood 2010;116:806-14.

[7] Tatsumi K, Mackman N. Tissue factor and atherothrombosis. J Atheroscler Thromb 2015;22:543-9.

[8] Drake TA, Morrissey JH, Edgington TS. Selective cellular expression of tissue factor in human tissues. Implications for disorders of hemostasis and thrombosis. Am J Pathol 1989;134:1087-97.

[9] Day SM, Reeve JL, Pedersen B, Farris DM, Myers DD, Im M, et al. Macrovascular thrombosis is driven by tissue factor derived primarily from the blood vessel wall. Blood 2005;105: 192-198.

[10] Wang L, Miller C, Swarthout RF, Rao M, Mackman N, Taubman MB. Vascular smooth muscle-derived tissue factor is critical for arterial thrombosis after ferric chloride-induced injury. Blood 2009;113:705-13.

[11] David TH, Sulciner DJ, Cornelissen I, Coughlin SR. Endothelial and hematopoietic tissue factor are unnecessary in two mouse models of hemostasis and thrombosis. Blood 2008;112: 3085.

[12] Pawlinski R, Mackman N. Cellular sources of tissue factor in endotoxemia and sepsis. Thromb Res 2010;125(Suppl. 1): S70-3.

[13] Chantrathammachart P, Mackman N, Sparkenbaugh E, Wang JG, Parise LV, Kirchhofer D, et al. Tissue factor promotes activation of coagulation and inflammation in a mouse model of sickle cell disease. Blood 2012;120:636-46.

[14] Sparkenbaugh EM, Chantrathammachart P, Mickelson J, van Ryn J, Hebbel RP, Monroe DM, et al. Differential contribution of FXa and thrombin to vascular inflammation in a mouse model of sickle cell disease. Blood 2014;123:1747-56. 